\title{
Synthesis and Antimicrobial Activity of Some New Piperazine Derivaties Containing Aryl Sulfonyloxy Group
}

\author{
H.S.PATEL*, H.D.DESAI and H.J.MISTRY \\ Department of Chemistry, S.P.University \\ V.V.Nagar -388 120, Gujarat (INDIA)
}

Received 3 March 2004; Accepted 20 March 2004

\begin{abstract}
Novel N-substituted piperazine derivatives containing sulfonyloxy aniline moiety have been prepared. The various 4-sulfonyloxy aniline (SA) derivatives (2a-h) have been prepared by the condensation reaction of N-Acetyl Sulfanilyl chloride (ASC) and sodium phenates followed by hydrolysis. The SA derivatives are then reacted with chloro acetyl chloride to give corresponding (N-Chloroacetyl) derivatives (3a-h). These derivatives are then reacted with $\mathrm{N}$-phenyl piperazine to yield the corresponding piperazine derivatives $(4 a-h)$.
\end{abstract}

Keywords: $N$-Phenyl piperazine, ASC, NMR spectroscopy

\section{Introduction}

Piperazine derivatives have been extensively investigated by the organic chemists due to their close association with various types of biological activities. Moreover they have wide clinical applications in the therapy of functional diseases and exhibit Anthelmintic, antibacterial and insecticidal activities ${ }^{1}$.Piperazine derivatives containing aryl $-\mathrm{SO}_{3}$ group has received no attention in spite of welldefined biological activities of piperazine containing compounds.Hence it was thought interesting to synthesize novel piperazine derivatives containing aryl $-\mathrm{SO}_{3}$ group. The present paper comprises the synthesis and characterization of piperazine derivatives as shown in Scheme -1.

Antibacterial activities:

Antibacterial activities of all the compounds were studied against Gram-positive bacteria (Bacillus subtillis and Staphylococcus aureus) and Gram-negative bacteria (E.coli and Salmonella typhi) at a concentration of $\tilde{\square} \square \mathrm{g} / \mathrm{ml}$ by Agar cup plate method. Methanol system was used as control in this method. Under similar condition using sulfanilamide as a standard for comparison carried out control experiment. The area of inhibition of zone is measured in $\mathrm{cm}$. Compounds $4 \mathrm{c}, 4 \mathrm{~d}$ and $4 \mathrm{~h}$ were found more active against the above microbes. Other compounds found to be less or moderate active than sulfanilamide (Table-2). 
<smiles>[R2]c1cc([R])c([N+](=O)[O-])c([R])c1</smiles><smiles>CC(=O)Nc1ccc(S(=O)(=O)Cl)cc1</smiles>

i) Acetone
ii) $\mathrm{NaHCO}_{3}$
iii) Pyridine<smiles>[R2]c1cc([R8])c(OS(=O)(=O)c2ccc(NC(C)=O)cc2)c([R8])c1</smiles>

$$
\begin{aligned}
& (1 \mathrm{a}-\mathrm{h}) \\
& \mathrm{Hydrolysis} \\
& \mathrm{EtOH} / \mathrm{HC}
\end{aligned}
$$<smiles>[R2]c1cc([R8])c(OS(=O)(=O)c2ccc(N)cc2)c([R3])c1</smiles>

$(2 \mathrm{a}-\mathrm{h})$

i) Phthalic anhydride

ii) Chloroform

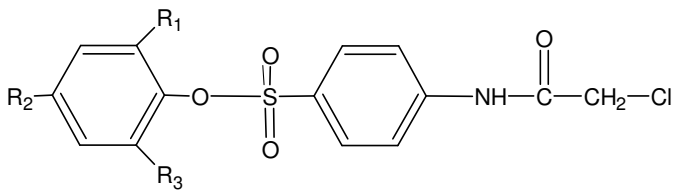

$(3 a-h)$
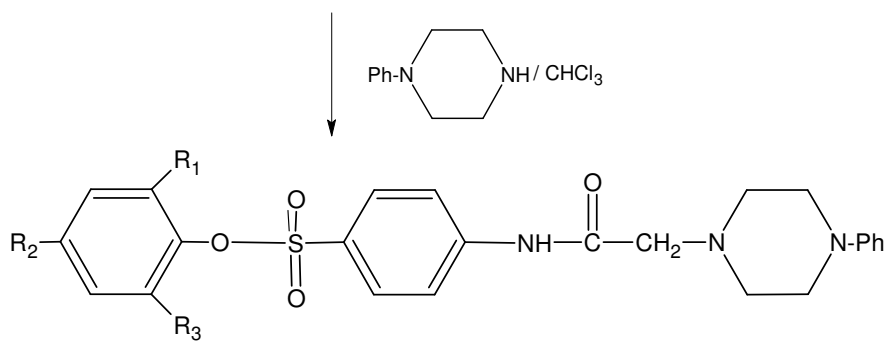

Scheme- 1 (4a-h)

\begin{tabular}{ccccccccc}
\hline Where & $\mathrm{a}$ & $\mathrm{b}$ & $\mathrm{c}$ & $\mathrm{d}$ & $\mathrm{E}$ & $\mathrm{f}$ & $\mathrm{g}$ & $\mathrm{h}$ \\
\hline $\mathrm{R}_{1}$ & $\mathrm{H}$ & $\mathrm{H}$ & $\mathrm{H}$ & $\mathrm{H}$ & $\mathrm{H}$ & $\mathrm{H}$ & $\mathrm{Cl}$ & $\mathrm{Br}$ \\
$\mathrm{R}_{2}$ & $\mathrm{H}$ & $\mathrm{CH}_{3}$ & $\mathrm{NO}_{2}$ & $\mathrm{Cl}$ & $\mathrm{OCH}_{3}$ & $\mathrm{C}\left(\mathrm{CH}_{3}\right)_{3}$ & $\mathrm{Cl}$ & $\mathrm{Br}$ \\
$\mathrm{R}_{3}$ & $\mathrm{H}$ & $\mathrm{H}$ & $\mathrm{H}$ & $\mathrm{H}$ & $\mathrm{H}$ & $\mathrm{H}$ & $\mathrm{H}$ & $\mathrm{Br}$ \\
\hline
\end{tabular}




\section{Ecperimental}

Melting points were determined in open capillary tube and are uncorrected. The IR spectra were recorded in $\mathrm{KBr}$ pallets on a Nicolet 400D spectrometer and ${ }^{1} \mathrm{H}$ NMR spectra in $\mathrm{CDCl}_{3}$ on Hitachi R1500, $60 \mathrm{MHz}$ spectrometer and Hitachi R- 1500, $60 \mathrm{MHz}$ spectrometer using TMS as an internal standard. The required 4- acetamidobenzene sulfonyl chloride (ASC) was prepared by reported $\operatorname{method}^{2}$. All the chemicals used were of laboratory grade.

Preparation of 4-(substituted phenyl sulfonyloxy) acetanilide (la-h)

Sodium salts of phenols (listed Scheme-1) (0.05 mole) was dissolved in a mixture of $40 \mathrm{ml}$ anhydrous acetone and $1 \mathrm{ml}$ of dry pyridine in $250 \mathrm{ml} \mathrm{R.B}$. flask and $11.67 \mathrm{gm}(0.05 \mathrm{~mole})$ of pure 4-acetamidobenzene sulfonyl chloride (ASC) was added portion wise with stirring. Sodium bicarbonate $(0.05$ mole) was added as an acid acceptor. The reaction mixture is set aside overnight and almost pure 4(substituted phenyl sulfonyloxy) acetanilide is filtered off and washed with cold water and air dried. It was then recrystallized from ethanol to give product (1a-h) in 60-70\% yield. All the compounds (1a-h) were then hydrolyzed as follows.

Preparation of $4-$ (substituted phenyl sulfonyloxy) aniline (2a-h) by hydrolysis of (1a-h) by hydrolysis of ( $1 a-h)$ Each of the compounds (1-ah) was hydrolyzed by refluxing with $75 \mathrm{ml}$ of ethanol containing $15 \mathrm{ml}$ of conc. $\mathrm{HCl}$ for 4-5 hrs. It was then poured into ice-cold water and finally made just alkaline with liq. ammonia. The resultant product 4-(substituted phenyl sulfonyloxy) aniline (2a-h) is filtered off and washed with water and dried. It was then recrystallized from ethanol give products ( $2 \mathrm{a}-\mathrm{h})$ in $65-75 \%$ yield. The details of all the compounds are furnished in Table- $\mathbf{1}$.

Preparation of $N$-chloroacetyl derivatives $(3 a-h)$

To a suspension of all the compound (2a-h) $(0.005$ mole), anhy. Potassium carbonate $(2.5 \mathrm{gm})$ and $1 \mathrm{ml}$ of TEA in dry chloroforms $(65 \mathrm{ml})$ was added chloro acetyl chloride $(0.006$ mole $)$ and the mixture was refluxed for 4-5 hrs. The solvent was then evaporated and the resulting solid was washed with cold water and recrystallized using appropriate solvent affording N-chloroacetyl derivatives (3a-h) in $60-$ $65 \%$ yield.

\section{Preparation of piperazine derivatives $(4 a-h)$}

To a solution of $\mathrm{N}$-chloroacetyl derivatives (3a-h) ( 0.005 mole) and $\mathrm{N}$-phenyl piperazine 0.005 mole in dry chloroform was refluxed for 1-2 hours. The solvent was evaporated and the resulting solid was washed with cold water. The solid thus obtained was purified by column chromatography over silica gel using ethyl acetate : benezene as eluent in appropriate proportions. Recrystallization from chloroform gave corresponding $(4 \mathrm{a}-\mathrm{h})$ in $50-60 \%$ yield.

\section{Results and Discussion}

The synthesis of piperazine derivatives is accomplished as shown in the Scheme-1. Analystcial and Spectral data are given in Table-2.

The compound ASC was reacted with various phenolates and followed by hydrolysis ${ }^{3}$. The resultant hydrolyzed products (2a-h) have consistent values of $\mathrm{C}, \mathrm{H}, \mathrm{N}$ and $\mathrm{S}$ contents with the predicted structure. All the compounds $(2 \mathrm{a}-\mathrm{h})$ show the diazo test for primary amine $\left(-\mathrm{NH}_{2}\right)$ group. The IR spectra of (2a-h) show the new bands at $3410 \mathrm{~cm}-1\left(\mathrm{NH}_{2}\right)$ and $1210-1150 \mathrm{~cm}^{-1}\left(\mathrm{SO}_{3}\right)$, than the IR spectra of ASC. The compounds (2a-h) then reacted with chloro acetyl chloride in chloroform to yield corresponding N-chloroacetyl derivatives ${ }^{4,5}(3 \mathrm{a}-\mathrm{h})$ show new IR spectral absorption bands at $1550 \mathrm{~cm}^{-}$ ${ }^{1}(-\mathrm{CONH})$. Further reaction of $(3 \mathrm{a}-\mathrm{h})$ with $\mathrm{N}$-phenyl piperazine afforded corresponding piperazine derivatives ${ }^{6}$. Their structures (4-ah) were confirmed by elemental analysis and IR spectral features and also confirms on the basis of ${ }^{1} \mathrm{H}$ NMR spectrum. 
Table 1. Analytical and Spectral data of compounds (2 a-h)

\begin{tabular}{|c|c|c|c|c|c|c|c|c|}
\hline \multirow[t]{2}{*}{ Compd } & \multirow{2}{*}{$\begin{array}{l}\text { Molecular } \\
\text { formula }\end{array}$} & \multicolumn{4}{|c|}{$\%$ Analysis Calculated (found) } & \multirow{2}{*}{$\begin{array}{c}\% \\
\text { Yield }\end{array}$} & \multirow{2}{*}{$\begin{array}{l}\text { M.P } \\
\left({ }^{0} \mathrm{C}\right)\end{array}$} & \multirow[t]{2}{*}{$\operatorname{PMR}(\delta$ ppm $)$} \\
\hline & & $\% \mathrm{C}$ & $\% \mathrm{H}$ & $\% \mathrm{~N}$ & $\% \mathrm{~S}$ & & & \\
\hline $2 \mathrm{a}$ & $\mathrm{C}_{12} \mathrm{H}_{11} \mathrm{O}_{3} \mathrm{NS}$ & $\begin{array}{c}57.83 \\
(57.65)\end{array}$ & $\begin{array}{c}4.41 \\
(4.35)\end{array}$ & $\begin{array}{c}5.62 \\
(5.45)\end{array}$ & $\begin{array}{c}12.85 \\
(12.60)\end{array}$ & 70 & 145 & $\begin{array}{lll}4.3(2 \mathrm{H}, \mathrm{s}, & \left.\mathrm{NH}_{2}\right), & 6.8-7.6(9 \mathrm{H}, \mathrm{m}, \\
\text { aromatic }) & & \end{array}$ \\
\hline $2 b$ & $\mathrm{C}_{13} \mathrm{H}_{13} \mathrm{O}_{3} \mathrm{NS}$ & $\begin{array}{c}59.31 \\
(59.20)\end{array}$ & $\begin{array}{c}4.94 \\
(4.75)\end{array}$ & $\begin{array}{c}5.32 \\
(5.20)\end{array}$ & $\begin{array}{c}12.16 \\
(12.95)\end{array}$ & 70 & 138 & $\begin{array}{l}2.2\left(3 \mathrm{H}, \mathrm{s}, \mathrm{CH}_{3}\right), 4.3\left(2 \mathrm{~h}, \mathrm{~s}, \mathrm{NH}_{2}\right), 6.5- \\
7.6(8 \mathrm{H}, \mathrm{m}, \text { aromatic })\end{array}$ \\
\hline $2 \mathrm{c}$ & $\mathrm{C}_{12} \mathrm{H}_{10} \mathrm{O}_{5} \mathrm{NSCl}$ & $\begin{array}{c}50.79 \\
(50.55)\end{array}$ & $\begin{array}{c}3.52 \\
(3.40)\end{array}$ & $\begin{array}{c}4.93 \\
(4.75)\end{array}$ & $\begin{array}{c}11.28 \\
(11.15)\end{array}$ & 65 & 120 & $\begin{array}{l}4.3\left(2 \mathrm{H}, \mathrm{s}, \quad \mathrm{NH}_{2}\right), \quad 6.5-7.8(9 \mathrm{H}, \mathrm{m}, \\
\text { aromatic })\end{array}$ \\
\hline $2 d$ & $\mathrm{C}_{12} \mathrm{H}_{10} \mathrm{O}_{5} \mathrm{~N}_{2} \mathrm{~S}$ & $\begin{array}{c}48.97 \\
(48.75)\end{array}$ & $\begin{array}{c}3.40 \\
(3.25)\end{array}$ & $\begin{array}{r}9.52 \\
(9.35)\end{array}$ & $\begin{array}{c}10.88 \\
(10.70)\end{array}$ & 80 & 105 & $\begin{array}{l}4.3\left(2 \mathrm{H}, \mathrm{s}, \quad \mathrm{NH}_{2}\right), \quad 6.8-7.8(9 \mathrm{H}, \mathrm{m}, \\
\text { aromatic })\end{array}$ \\
\hline $2 \mathrm{e}$ & $\mathrm{C}_{13} \mathrm{H}_{13} \mathrm{O}_{4} \mathrm{NS}$ & $\begin{array}{c}55.91 \\
(55.75)\end{array}$ & $\begin{array}{c}4.65 \\
(4.50)\end{array}$ & $\begin{array}{c}5.01 \\
(4.85)\end{array}$ & $\begin{array}{c}11.40 \\
(11.30)\end{array}$ & 75 & 125 & $\begin{array}{l}3.8\left(3 \mathrm{H}, \mathrm{s}, \mathrm{OCH}_{3}\right), 4.3\left(2 \mathrm{H}, \mathrm{s}, \mathrm{NH}_{2}\right), \\
6.4-7.5(8 \mathrm{H}, \mathrm{m}, \text { aromatic })\end{array}$ \\
\hline $2 \mathrm{f}$ & $\mathrm{C}_{16} \mathrm{H}_{19} \mathrm{O}_{3} \mathrm{NS}$ & $\begin{array}{c}62.95 \\
(62.80)\end{array}$ & $\begin{array}{c}6.22 \\
(6.05)\end{array}$ & $\begin{array}{c}4.59 \\
(4.45)\end{array}$ & $\begin{array}{c}10.49 \\
(10.35)\end{array}$ & 60 & 118 & $\begin{array}{l}2.2\left(9 \mathrm{H}, \mathrm{s}, \quad 3 \mathrm{CH}_{3}\right), 4.2\left(2 \mathrm{H}, \mathrm{s}, \mathrm{NH}_{2}\right), \\
6.4-7.8(8 \mathrm{H}, \mathrm{m}, \text { aromatic })\end{array}$ \\
\hline $2 \mathrm{~g}$ & $\mathrm{C}_{12} \mathrm{H}_{9} \mathrm{O}_{3} \mathrm{NSCl}_{2}$ & $\begin{array}{r}45.28 \\
(45.10)\end{array}$ & $\begin{array}{c}2.83 \\
(2.65)\end{array}$ & $\begin{array}{c}4.40 \\
(4.20)\end{array}$ & $\begin{array}{l}10.06 \\
(9.85)\end{array}$ & 75 & 150 & $\begin{array}{l}4.4\left(2 \mathrm{H}, \mathrm{s}, \quad \mathrm{NH}_{2}\right), \quad 6.8-8.0 \quad(7 \mathrm{H}, \mathrm{m}, \\
\text { aromatic })\end{array}$ \\
\hline $2 \mathrm{~h}$ & $\mathrm{C}_{12} \mathrm{H}_{8} \mathrm{O}_{3} \mathrm{NS} \mathrm{Br}_{3}$ & $\begin{array}{c}29.62 \\
(29.40)\end{array}$ & $\begin{array}{c}1.64 \\
(1.50)\end{array}$ & $\begin{array}{c}2.88 \\
(2.65)\end{array}$ & $\begin{array}{c}6.58 \\
(6.45)\end{array}$ & 72 & 155 & $\begin{array}{ll}4.4(2 \mathrm{H}, \mathrm{s}, & \left.\mathrm{NH}_{2}\right), 6.8-8.0 \quad(6 \mathrm{H}, \mathrm{m}, \\
\text { aromatic }) & \end{array}$ \\
\hline
\end{tabular}


Table - 2. Analytical and Spectral data of Compounds (4a-h)

\begin{tabular}{|c|c|c|c|c|c|c|c|c|}
\hline \multirow[b]{2}{*}{ Comps } & \multirow{2}{*}{$\begin{array}{l}\text { Molecular } \\
\text { formula }\end{array}$} & \multicolumn{4}{|c|}{$\%$ Analysis Calculated (Found) } & \multirow{2}{*}{$\begin{array}{l}\text { Yield } \\
(\%)\end{array}$} & \multirow{2}{*}{$\begin{array}{l}\text { M.P. } \\
\left({ }^{0} \mathrm{C}\right)\end{array}$} & \multirow{2}{*}{$\operatorname{PMR}(\delta, p p m)$} \\
\hline & & $\% \mathrm{C}$ & $\% \mathrm{H}$ & $\% \mathrm{~N}$ & $\% \mathrm{~S}$ & & & \\
\hline $4 a$ & $\mathrm{C}_{24} \mathrm{H}_{25} \mathrm{O}_{4} \mathrm{~N}_{3} \mathrm{~S}$ & $\begin{array}{l}63.85 \\
(63.75)\end{array}$ & $\begin{array}{c}5.54 \\
(5.45)\end{array}$ & $\begin{array}{l}9.31 \\
(9.20)\end{array}$ & $\begin{array}{c}7.09 \\
(6.90)\end{array}$ & 170 & 55 & $\begin{array}{l}\left.\text { 2.2-3.1(10H, t+s, } 5 \mathrm{CH}_{2}\right), \\
\text { 6.5-7.6 (14H,m, aromatic), } \\
\text { 9.2(1H,hump,NH, })\end{array}$ \\
\hline $4 b$ & $\mathrm{C}_{25} \mathrm{H}_{27} \mathrm{O}_{4} \mathrm{~N}_{3} \mathrm{~S}$ & $\begin{array}{c}64.51 \\
(64.40)\end{array}$ & $\begin{array}{c}5.80 \\
(5.70)\end{array}$ & $\begin{array}{c}9.03 \\
(8.90)\end{array}$ & $\begin{array}{c}6.88 \\
(6.75)\end{array}$ & 165 & 60 & $\begin{array}{l}\text { 1.8 }\left(3 \mathrm{H}, \mathrm{s}, \mathrm{CH}_{3}\right), \\
\left.\text { 2.2-3.2(10H,t+s, } 5 \mathrm{CH}_{2}\right), \\
\text { 6.5-7.6 }(13 \mathrm{H}, \mathrm{m}, \text { aromatic }) \text {, } \\
\text { 9.2(1H,hump,NH, })\end{array}$ \\
\hline $4 c$ & $\mathrm{C}_{24} \mathrm{H}_{24} \mathrm{O}_{6} \mathrm{~N}_{3} \mathrm{~S}$ & $\begin{array}{c}58.06 \\
(57.95)\end{array}$ & $\begin{array}{c}4.83 \\
(4.75)\end{array}$ & $\begin{array}{c}11.29 \\
(11.10 \\
)\end{array}$ & $\begin{array}{c}6.45 \\
(6.35)\end{array}$ & 205 & 53 & $\begin{array}{l}\text { 2.3-3.1 }\left(10 \mathrm{H}, \mathrm{t}+\mathrm{s}, 5 \mathrm{CH}_{2}\right), \\
\text { 6.5-7.9 (13H,m, aromatic), } \\
\text { 9.2(1H,hump,NH, })\end{array}$ \\
\hline $4 d$ & $\begin{array}{c}\mathrm{C}_{24} \mathrm{H}_{24} \mathrm{O}_{4} \mathrm{~N}_{3} \mathrm{~S} \\
\mathrm{Cl}\end{array}$ & $\begin{array}{c}59.32 \\
(59.25)\end{array}$ & $\begin{array}{c}4.94 \\
(4.80)\end{array}$ & $\begin{array}{c}8.65 \\
(8.50)\end{array}$ & $\begin{array}{c}6.59 \\
(6.50)\end{array}$ & 155 & 50 & $\begin{array}{l}\left.\text { 2.3-3.2(10H,t+s, } 5 \mathrm{CH}_{2}\right), \\
\text { 6.9-8.2 (13H,m, aromatic), } \\
\text { 9.3(1H,hump,NH, })\end{array}$ \\
\hline $4 \mathrm{e}$ & $\mathrm{C}_{25} \mathrm{H}_{27} \mathrm{O}_{5} \mathrm{~N}_{3} \mathrm{~S}$ & $\begin{array}{c}62.37 \\
(62.30)\end{array}$ & $\begin{array}{c}5.61 \\
(5.50)\end{array}$ & $\begin{array}{c}8.73 \\
(8.65)\end{array}$ & $\begin{array}{c}6.65 \\
(6.55)\end{array}$ & 175 & 55 & $\begin{array}{l}\text { 2.3-3.2 }\left(10 \mathrm{H}, \mathrm{t}+\mathrm{s}, 5 \mathrm{CH}_{2}\right), \\
\text { 3.6 }\left(3 \mathrm{H}, \mathrm{S}, \mathrm{OCH}_{3}\right) \\
\text { 6.9-8.1 }(13 \mathrm{H}, \mathrm{m}, \text { aromatic }) \text {, } \\
\text { 9.3(1H,hump,NH, }\end{array}$ \\
\hline $4 f$ & $\mathrm{C}_{28} \mathrm{H}_{33} \mathrm{O}_{4} \mathrm{~N}_{3} \mathrm{~S}$ & $\begin{array}{c}66.27 \\
(66.15)\end{array}$ & $\begin{array}{c}6.50 \\
(6.40)\end{array}$ & $\begin{array}{c}8.28 \\
(8.15)\end{array}$ & $\begin{array}{c}6.31 \\
(6.20)\end{array}$ & 167 & 52 & $\begin{array}{l}1.3\left(9 \mathrm{H}, \mathrm{s}, 3 \mathrm{CH}_{3}\right), \\
2.2-3.1\left(10 \mathrm{H}, \mathrm{t}+\mathrm{s}, 5 \mathrm{CH}_{2}\right), \\
6.8-8.1(13 \mathrm{H}, \mathrm{m}, \text { aromatic }) \text {, } \\
\text { 9.2(1H,hump,NH, }\end{array}$ \\
\hline $4 \mathrm{~g}$ & $\begin{array}{c}\mathrm{C}_{25} \mathrm{H}_{23} \mathrm{O}_{4} \mathrm{~N}_{3} \\
\mathrm{Cl}_{2} \mathrm{~S}\end{array}$ & $\begin{array}{c}55.49 \\
(55.40)\end{array}$ & $\begin{array}{c}4.43 \\
(4.30)\end{array}$ & $\begin{array}{c}8.09 \\
(7.90)\end{array}$ & $\begin{array}{l}6.16 \\
(6.05)\end{array}$ & 177 & 54 & $\begin{array}{l}\left.\text { 2.2-3.3(10H,t+s, } 5 \mathrm{CH}_{2}\right), \\
\text { 6.5-8.1 (12H,m, aromatic), } \\
\text { 9.1(1H,hump,NH, })\end{array}$ \\
\hline $4 \mathrm{~h}$ & $\begin{array}{c}\mathrm{C}_{24} \mathrm{H}_{22} \mathrm{O}_{4} \mathrm{~N}_{3} \mathrm{~S} \\
\mathrm{Br}_{3}\end{array}$ & $\begin{array}{c}46.15 \\
(46.05)\end{array}$ & $\begin{array}{c}3.52 \\
(3.45)\end{array}$ & $\begin{array}{c}6.73 \\
(6.65)\end{array}$ & $\begin{array}{c}5.12 \\
(0.05)\end{array}$ & 148 & 55 & $\begin{array}{l}\text { 2.3-3.1 }\left(10 \mathrm{H}, \mathrm{t}+\mathrm{s}, 5 \mathrm{CH}_{2}\right), \\
\text { 6.8-8.3 }(11 \mathrm{H}, \mathrm{m}, \text { aromatic }) \text {, } \\
\text { 9.2(1H,hump,NH, })\end{array}$ \\
\hline
\end{tabular}


Table 3. Antibacterial Activity of compounds (4a-h)

\begin{tabular}{ccccc}
\hline \multirow{2}{*}{ Compounds } & \multicolumn{3}{c}{ Zone of inhibition } \\
& \multicolumn{2}{c}{ Gram +ve } & \multicolumn{2}{c}{ Gram -ve } \\
\cline { 2 - 5 } & Bacillus & Staphylococcus & Salmonella \\
Subtillis & Aureus & E.Coli \\
\hline 4a & 55 & 60 & 43 & 65 \\
4b & 42 & 67 & 52 & 69 \\
4c & 70 & 80 & 75 & 60 \\
4d & 83 & 70 & 68 & 55 \\
4e & 45 & 65 & 43 & 83 \\
4f & 65 & 60 & 62 & 49 \\
4g & 68 & 55 & 68 & 58 \\
4h & 83 & 69 & 79 & 72 \\
Sulfanilamide & 85 & 65 & 75 & 73 \\
\hline
\end{tabular}

\section{Acknowledgement}

The authors are thankful to Dr. R.M. Patel, Head, Department of Chemistry for providing laboratory facilities.

\section{References}

1. Coyne W E Medicinal chemistry, edited by Alfred burger (Wiley- Interscience, New Your), 1970

2. Vogel A I A Textbook of Practical Organic Chemistry, 4th ed., 1978, 651.

3. Vogel A I A Textbook of Practical Organic Chemistry, 4th ed., 1978, 652 -653.

4. Clark N G and Hams A F Biochem. J. 1953, 55, 839.

5. Arya V P, Grewal R S, Kavl C L ,David J and Honkan V Indian J. Chem. 1977, 15B, 133.

6. Holinska Hana, Protiva Miroslav and Frycova Hana Chem. Abstr. 1990, 113, 59226h. 


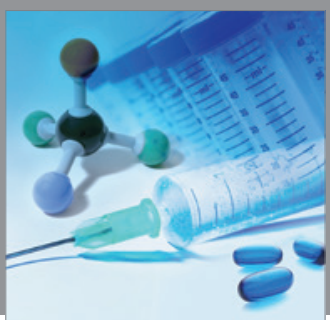

International Journal of

Medicinal Chemistry

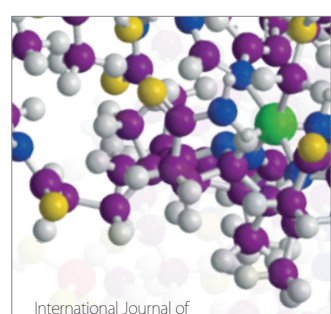

Carbohydrate Chemistry

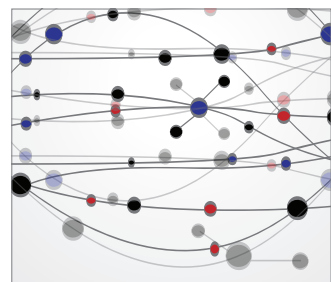

The Scientific World Journal
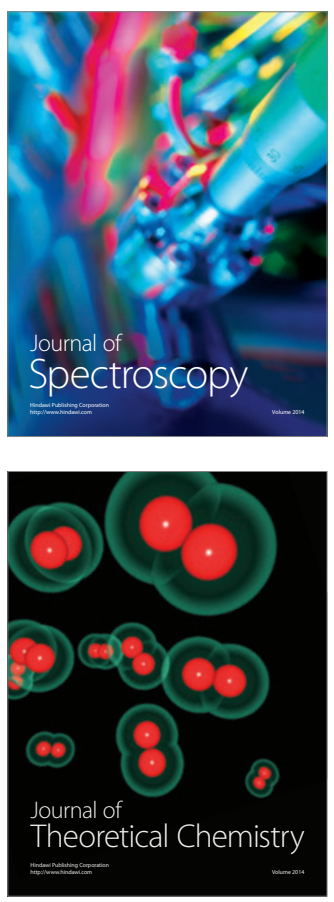
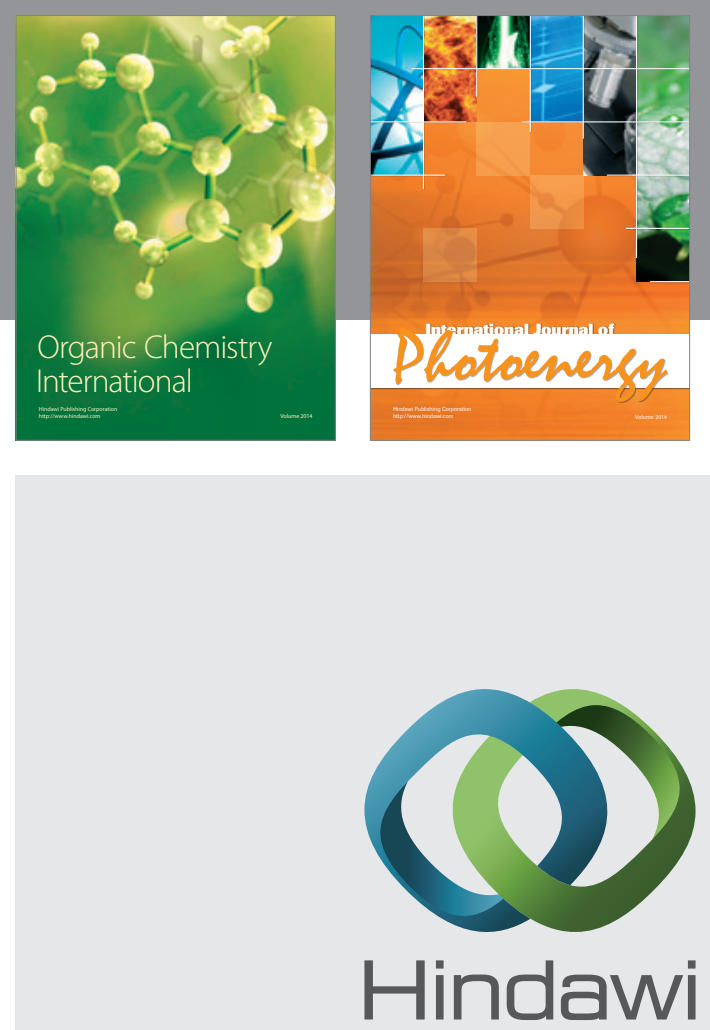

Submit your manuscripts at

http://www.hindawi.com
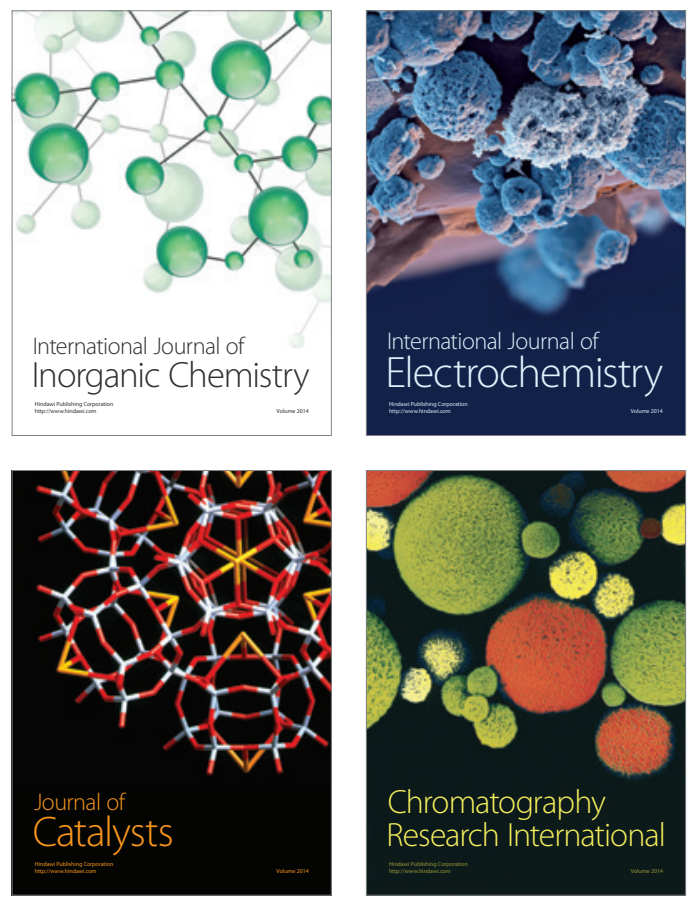
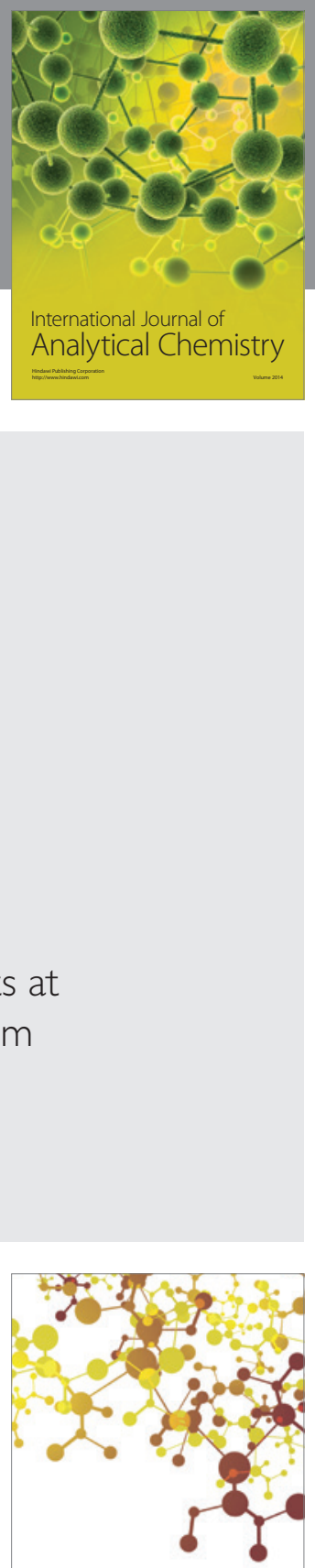

Journal of

Applied Chemistry
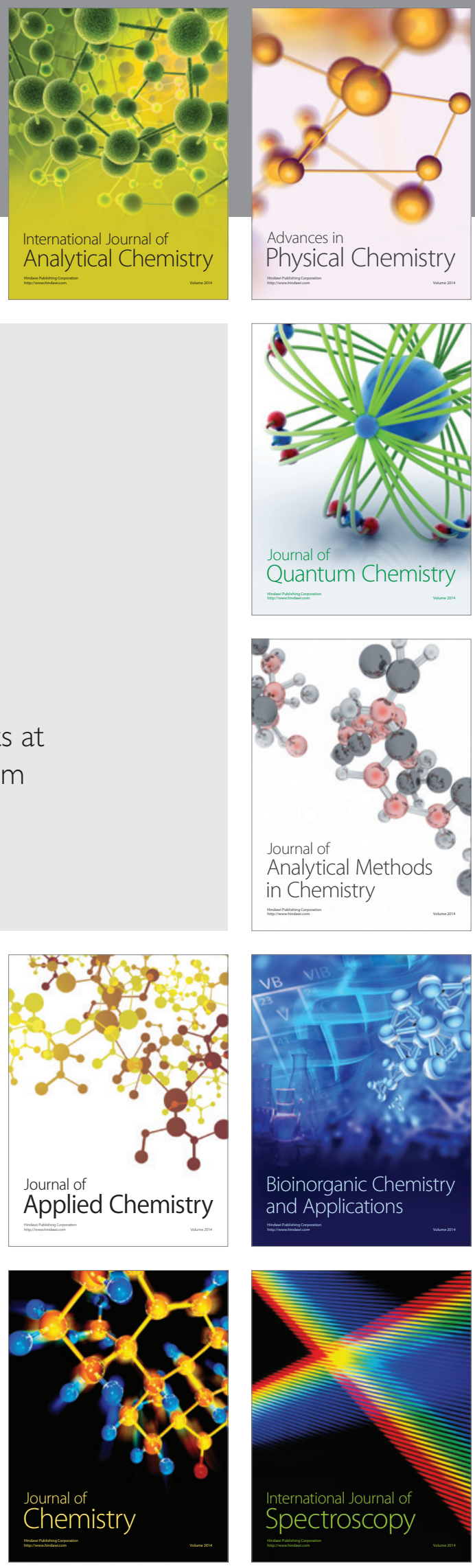